\title{
Relativistic effects on magnetic circular dichroism studied by GUHF/SECI method
}

\author{
Y. Honda $^{\text {a }}$, M. Hada ${ }^{\text {a }}$, M. Ehara ${ }^{\text {a }}$, H. Nakatsuji ${ }^{\text {a,* }}$, J. Downing ${ }^{\text {b }}$, J. Michl ${ }^{\text {b }}$ \\ a Department of Synthetic Chemistry and Biological Chemistry, Graduate School of Engineering, Kyoto University, Sakyo-ku, \\ Kyoto 606-8501, Japan \\ ${ }^{\mathrm{b}}$ Department of Chemistry and Biochemistry, University of Colorado, Boulder, CO 80309-0215, USA
}

Received 26 November 2001; in final form 27 December 2001

\begin{abstract}
Quasi-relativistic formulation of the Magnetic circular dichroism (MCD) Faraday terms are presented using the generalized unrestricted Hartree-Fock (GUHF)/single excitation configuration interaction (SECI) method combined with the finite perturbation method and applied to the MCD of the three $\mathrm{n}-\sigma^{*}$ states $\left({ }^{3} \mathrm{Q}_{1},{ }^{3} \mathrm{Q}_{0},{ }^{1} \mathrm{Q}_{1}\right)$ of $\mathrm{CH}_{3} \mathrm{I}$. The Faraday $B$ term for the ${ }^{1} \mathrm{Q}_{1}$ state was 0.1976 (Debye $)^{2}$ (Bohr magneton) $/\left(10^{3} \mathrm{~cm}^{-1}\right)$ in the non-relativistic theory, but was dramatically improved by the relativistic effect and became 0.0184 in agreement with the experimental values, 0.014 and 0.0257 . This change was mainly due to the one-electron spin-orbit (SO1) term rather than the spin-free relativistic (SFR) and the two-electron spin-orbit (SO2) terms. (c) 2002 Elsevier Science B.V. All rights reserved.
\end{abstract}

\section{Introduction}

Magnetic circular dichroism (MCD) is caused by the difference of absorption intensities between dextrorotatory and levorotatory circular polarized light when an external magnetic field is applied to a molecular system, and is measured as MCD ellipticity. While absorption intensities are always positive, ellipticities can have both positive and negative values. Therefore, MCD is particularly useful for assignment of plural overlapping elec-

\footnotetext{
${ }^{*}$ Corresponding author. Fax: +81-75-753-5910.

E-mail address: hiroshi@subchem.kyoto-u.ac.jp (H. Nakatsuji).
}

tronic bands and vibronic bands. The MCD spectra of simple polyatomic molecules were observed for acetylene [1], ethylene [2,3], tetramethylethylene [3], cyclopropane [4], benzene derivatives [5-9], quinones [10], and so on.

The theory of MCD was established by 1970s [11]. Since then many theoretical studies have been published [10,12-19]. For example, Shieh et al. [12] reported the theory for MCD of molecules in dense media, and Coriani et al. [13] reported the MCD formulation for coupled cluster response theory. The calculations of MCD with the molecular orbital theory have been applied to ethylene [14], cyclopropane [15], formaldehyde [16], benzene derivatives $[17,18]$, quinones $[10,14]$, and many aromatic [18] and non-aromatic [19] cyclic 
$\pi$-electron compounds. Semi-empirical methods were employed in these calculations except for $[14,15]$, in which ab initio methods were employed. Sum-over-states (SOS) method was used in the calculations of the Faraday $B$ term in almost all of the previous studies except for the works by Coriani et al. [14] and Seamans and Linderberg [17]. The SOS method, however, has three disadvantages, i.e., being time-consuming, dependent on the number of the intermediate states used after truncation, and inaccuracy intrinsic to the SOS method even if all the intermediate states are considered [20] except for the case of full CI. These disadvantages can be solved by using quadratic response theory [14] or finite perturbation method [17]. We employed the finite perturbation method, and the details will be published in the near future.

The relativistic effect on the MCD have not yet been considered explicitly even though the MCD spectra for heavy systems have been observed for halogen molecules [21], methyl halides [22,23], and carbon tetrahalides [24]. Although the spin-orbit (SO) interaction was considered in $[21,24]$, the SO terms were merely treated as parameters. In order to investigate the relativistic effects in MCD, we have developed quasi-relativistic generalized unrestricted Hartree-Fock (GUHF) [25,26]/single excitation configuration interaction (SECI) method. In this Letter, we apply this method to methyl iodide $\left(\mathrm{CH}_{3} \mathrm{I}\right)$, for which the relativistic effects are expected to be large. Although one band is seemingly observed at the energy region of $30000-48000 \mathrm{~cm}^{-1}$ in the absorption spectrum of $\mathrm{CH}_{3} \mathrm{I}$, it is inconsistent with the shape of the MCD spectrum [22,23]. It is plausible that this band consists of three $\mathrm{n}-\sigma^{*}$ excited states. These are called ${ }^{3} \mathrm{Q}_{1},{ }^{3} \mathrm{Q}_{0}$, and ${ }^{1} \mathrm{Q}_{1}$ states, in the energy increasing order, according to Mulliken's notation [27]. The ${ }^{3} \mathrm{Q}_{1}$ and ${ }^{3} \mathrm{Q}_{0}$ states correspond to triplet states in the non-relativistic limit and have transition intensities through the SO interactions with the singlet states. This system is thus suitable for investigating the relativistic effects. We will see that the Faraday term of the ${ }^{1} Q_{1}$ state is much improved by the relativistic effect. The relativistic effects are expected to be very important for the MCD of the systems containing heavy atoms.

\section{Theory and calculational method}

The MCD ellipticity $\theta$ is proportional to the strength of an external magnetic field $H$ if $H$ is small enough. The ellipticity per unit magnetic field is strictly defined as derivative of $\theta$ with respect to $H$ in the limit of $H \rightarrow 0$ and is characterized by three parameters $A, B, C$ and one shape function $f\left(\omega_{j a}, \omega\right)$ assumed for spectral shapes [11], namely,

$$
\begin{aligned}
\left(\frac{\partial \theta}{\partial H}\right)_{H=0}= & -N \frac{2 \pi^{2}}{3 c \hbar}\left[\left(-\hbar^{-1}\right)\left(\frac{\partial f\left(\omega_{j a}, \omega\right)}{\partial \omega}\right)_{\omega_{j a}^{0}}\right. \\
& \left.\cdot A+f\left(\omega_{j a}, \omega\right)\left(B+\frac{C}{k T}\right)\right],
\end{aligned}
$$

where $N$ and $k T$ denote the number of the molecules in the light path and the Boltzmann factor, respectively. The three parameters are called Faraday $A, B$, and $C$ terms and are given by

$$
\begin{aligned}
A= & \frac{1}{2 d_{a}} \sum_{j \leftarrow a}\left[\left(\boldsymbol{\mu}_{j j}-\boldsymbol{\mu}_{a a}\right) \cdot \operatorname{Im}\left(\boldsymbol{m}_{a j} \times \boldsymbol{m}_{j a}\right)\right], \\
B= & \frac{1}{d_{a}} \sum_{j \leftarrow a} \operatorname{Im}\left[\sum_{n \neq a} \frac{\boldsymbol{\mu}_{n a} \cdot\left(\boldsymbol{m}_{a j} \times \boldsymbol{m}_{j n}\right)}{E_{n}-E_{a}}\right. \\
& \left.+\sum_{n \neq j} \frac{\boldsymbol{\mu}_{j n} \cdot\left(\boldsymbol{m}_{a j} \times \boldsymbol{m}_{n a}\right)}{E_{n}-E_{j}}\right], \\
C= & \frac{1}{2 d_{a}} \sum_{j \leftarrow a}\left[\boldsymbol{\mu}_{a a} \cdot \operatorname{Im}\left(\boldsymbol{m}_{a j} \times \boldsymbol{m}_{j a}\right)\right],
\end{aligned}
$$

where $d_{\mathrm{a}}$ is degeneracy of the ground state $a$ (while indices $j$ and $n$ stand for the excited states), and $\boldsymbol{m}$ and $\boldsymbol{\mu}$ are the electric and magnetic (transition) dipole moments, respectively. $\boldsymbol{m}_{a j}$ means $\langle a|\boldsymbol{m}| j\rangle$, for example, and Im denotes imaginary part. The summations over $j \leftarrow a$ means the sum over all the degenerate substates of $a$ and $j$. If neither of $a$ nor $j$ is degenerate, we can ignore these summations.

The Faraday $B$ term may be described differently from the sum-over-states form of Eq. (3). The $B$ term in $z$-direction, $B(z)$, is expressed as

$B(z)=\frac{3}{2 d_{a}} \sum_{j \leftarrow a}\left[\frac{\partial}{\partial H_{z}} \operatorname{Im}\left(\boldsymbol{m}_{a j} \times \boldsymbol{m}_{j a}\right)_{z}\right]_{H_{z}=0}$. 
The isotropically averaged $B$ term is given by

$$
\begin{aligned}
B & =\frac{B(x)+B(y)+B(z)}{3} \\
& =\frac{1}{2 d_{a}} \sum_{\alpha \beta \gamma} \varepsilon_{\alpha \beta \gamma} \sum_{j \leftarrow a}\left[\frac{\partial}{\partial H_{\gamma}} \operatorname{Im}\left(m_{a j, \alpha}^{(\gamma)} m_{j a, \beta}^{(\gamma)}\right)\right]_{H_{\gamma}=0},
\end{aligned}
$$

where $\varepsilon$ is Levi-Civata tensor. The symbols $\alpha, \beta, \gamma$ run over $x, y, z$, and $m_{\alpha}^{(\gamma)}$ is the $\alpha$-direction component of $\boldsymbol{m}$ in a $\gamma$-direction magnetic field, $H_{\gamma}$. We actually obtained the $B$ term by numerical differentiation of Eq. (6). The $B$ term expressed by Eqs. (5) and (6) is superior to that by Eq. (3) as mentioned in Section 1.

In order to calculate the $B$ term defined as given by Eqs. (5) and (6), we need the wavefunction in the finite magnetic fields. Such wavefunctions can be obtained by GUHF/SECI method. The $N$ electron GUHF wavefunction is given by a Slater determinant,

$\Psi_{\mathrm{GUHF}}=\left\|\psi_{1} \psi_{2} \cdots \psi_{j} \cdots \psi_{N}\right\|$,

where the molecular orbital, $\psi_{j}$, is described by a general spin orbital,

$\psi_{j}=\psi_{j}^{\alpha} \alpha+\psi_{j}^{\beta} \beta$.

The GUHF wavefunction is adequate for the relativistic Hamiltonian, being appropriately responsive to the magnetic field and the SO interactions, and therefore, it gives the best expression of the relativistic ground state in a magnetic field within the HF approximation. The SECI wavefunction describes the excited states and is defined by

$\Psi_{\mathrm{GUHF}-\mathrm{SECI}}=\left(\sum_{i}^{\mathrm{occ}} \sum_{a}^{\text {unocc }} C_{i}^{a} \hat{t}_{i}^{a}\right) \Psi_{\mathrm{GUHF}}$,

where $t_{i}^{a}=a_{a}^{\dagger} a_{i}$ represents an excitation operator from occupied orbital $i$ to unoccupied orbital $a$, and $C_{i}^{a}$ is its CI coefficient. The relativistic corrections and the response to the magnetic fields are considered at the SCF level and are transferred to the SECI wavefunctions through the reference function, $\Psi_{\mathrm{GUHF}}$. Although the SECI approximation gives rather poor excitation energies as compared with the symmetry adapted cluster-CI (SAC-CI) method [28], for example, the MCD
Faraday terms are expected to be qualitatively valid, because they essentially reflect one-electron processes.

We employ a quasi-relativistic (2-component) method for the relativistic GUHF/SECI calculations. The Hamiltonian for full-relativistic (Full-Rel) calculations was constructed by the one-electron second-order Douglas-Kroll transformation [29] and included the Breit-Pauli type two-electron spin-orbit (SO2) terms. For comparison, non-relativistic (Non-Rel), spin-free relativistic (SFR) terms only, one-electron spin-orbit (SO1) terms only, and SFR + SO1 calculations were also performed. The perturbation due to the magnetic fields was considered to first-order and its relativistic correction was not included in the present calculations. The molecule calculated was methyl iodide $\left(\mathrm{CH}_{3} \mathrm{I}\right)$, and the geometry was taken from the microwave and infrared measurements [30]. The basis set was Huzinaga's all-primitive (43222/4222/42) plus his d-polarization functions [31] for I, and Huzinaga-Dunning's (9s5p/4s)/ [4s $2 \mathrm{p} / 2 \mathrm{~s}]$ with standard scaling factors [32] plus Huzinaga's d-polarization functions [31] for $\mathrm{C}$ and $\mathrm{H}$ atoms. For relativistic calculations, we also used Huzinaga's (28s23p17d)/[11s8p6d] well-tempered Gaussian (WTG) basis set [33] contracted using the second-order Douglas-Kroll calculations for I, and Huzinaga-Dunning's $(9 \mathrm{~s} 5 \mathrm{p} / 4 \mathrm{~s}) /[5 \mathrm{~s} 3 \mathrm{p} / 3 \mathrm{~s}]$ plus their the first-order basis functions (FOBFs) [34] for $\mathrm{C}$ and $\mathrm{H}$, respectively. The inner core orbitals of $\mathrm{I}$ and $\mathrm{C}$ were frozen in the SECI calculations. The gauge origin was taken at the center of gravity of the nuclei. The gauge dependence of the Faraday terms was also examined, and we confirmed that the dependence is negligible for the present system.

\section{Results and discussion}

Table 1 shows the SECI excitation energies, MCD Faraday $A$ and $B$ terms, dipole strengths and oscillator strengths of the three excited states, ${ }^{3} \mathrm{Q}_{1},{ }^{3} \mathrm{Q}_{0}$, and ${ }^{1} \mathrm{Q}_{1}$, of $\mathrm{CH}_{3} \mathrm{I}$ calculated by the NonRel and Full-Rel GUHF/SECI methods. The experimental values by Gedanken and Rowe [22] and Johnson and Kinsey [23] are listed in the table. The 
Table 1

Excitation energies, Faraday $A$ terms $(A)$, Faraday $B$ terms $(B)$, dipole strengths $(D)$, and oscillator strengths $(f)$ for the ${ }^{3} \mathrm{Q}_{1},{ }^{3} \mathrm{Q}_{0}$, and ${ }^{1} \mathrm{Q}_{1}$ states of $\mathrm{CH}_{3} \mathrm{I}$ calculated by GUHF/SECI

\begin{tabular}{|c|c|c|c|c|c|c|}
\hline & \multicolumn{3}{|c|}{ GUHF/SECI } & \multirow{2}{*}{$\frac{\mathrm{SAC}-\mathrm{CI}}{\mathrm{SO} 1(\mathrm{HUZ})^{\mathrm{a}}}$} & \multicolumn{2}{|l|}{ Exptl. } \\
\hline & $\begin{array}{l}\text { Non-Rel } \\
(\text { HUZ) })^{\mathrm{a}}\end{array}$ & $\begin{array}{l}\text { Full-Rel } \\
\left(^{\text {HUZ) }}{ }^{\mathrm{a}}\right.\end{array}$ & $\begin{array}{l}\text { Full-Rel } \\
\text { (WTG) }^{\mathrm{b}}\end{array}$ & & Gedanken et al. ${ }^{\mathrm{c}}$ & Johnson et al. $^{\mathrm{d}}$ \\
\hline $\begin{array}{l}{ }^{3} \mathrm{Q}_{1} \text { state } \\
\text { energy }\end{array}$ & 40252 & 39188 & 38368 & 37300 & 33350 & 34900 \\
\hline$A^{\mathrm{f}}$ & 0.0 & -0.0140 & -0.0025 & & -0.0015 & $(-0.0097)^{\mathrm{g}}$ \\
\hline$B^{\mathrm{h}}$ & 0.0 & 0.1778 & 0.0746 & & 0.0018 & -0.000057 \\
\hline$D^{\mathrm{i}}$ & 0.0 & 0.0267 & 0.0048 & & 0.0029 & 0.0193 \\
\hline$f$ & 0.0 & 0.00049 & 0.000088 & & 0.000046 & 0.00032 \\
\hline $\begin{array}{l}{ }^{3} \mathrm{Q}_{0} \text { state } \\
\text { energy }\end{array}$ & 40252 & 38326 & 37506 & 40200 & 38300 & 38900 \\
\hline$A^{\mathrm{f}}$ & j & - & - & & - & - \\
\hline$B^{\mathrm{h}}$ & 0.0 & -0.1453 & -0.0655 & & -0.015 & -0.0222 \\
\hline$D^{\mathrm{i}}$ & 0.0 & 0.3629 & 0.3715 & & 0.32 & 0.284 \\
\hline$f$ & 0.0 & 0.0065 & 0.0066 & & 0.0057 & 0.0051 \\
\hline $\begin{array}{l}{ }^{1} Q_{1} \text { state } \\
\text { energy }\end{array}$ & 48226 & 47850 & 46905 & 43500 & 41700 & 40900 \\
\hline$A^{\mathrm{f}}$ & -0.2394 & -0.0756 & -0.0092 & & -0.044 & $(-0.0377)^{\mathrm{g}}$ \\
\hline$B^{\mathrm{h}}$ & 0.1976 & 0.0184 & 0.0073 & & 0.014 & 0.0257 \\
\hline$D^{\mathrm{i}}$ & 0.4893 & 0.1257 & 0.0167 & & 0.088 & 0.0753 \\
\hline$f$ & 0.0111 & 0.0028 & 0.00037 & & 0.0017 & 0.0014 \\
\hline
\end{tabular}

${ }^{a}$ Huzinaga's uncontracted basis set with exponents determined by a non-relativistic method.

${ }^{\mathrm{b}}$ Huzinaga's well-tempered Gaussian basis set contracted using the second-order Douglas-Kroll SCF calculations.

${ }^{\mathrm{c}}[22]$.

${ }^{d}$ [23]. Reassignments of the absorption and MCD spectra in [22].

${ }^{\mathrm{e}}$ Unit of $\mathrm{cm}^{-1}$.

${ }^{\mathrm{f}}$ Unit of (Debye) $)^{2}$ (Bohr magneton).

${ }^{\mathrm{g}}$ Assumed the magnetic moment to be 1 Bohr magneton.

${ }^{\mathrm{h}}$ Unit of $10^{-3}$ (Debye) $)^{2}$ (Bohr magneton) $/ \mathrm{cm}^{-1}$.

${ }^{\mathrm{i}}$ Unit of (Debye) ${ }^{2}$.

${ }^{\mathrm{j}}$ Owing to the non-degeneracy, $A$ terms disappear.

unit of each property is given in the footnote. Since the ground state is non-degenerate, the Faraday $C$ term does not appear. For comparison between Non-Rel and Full-Rel calculations, we mainly discuss the results with Huzinaga's uncontracted non-relativistic basis.

For the ${ }^{1} \mathrm{Q}_{1}$ state, the $B$ term is significantly improved from the Non-Rel value, 0.1976 $(\text { Debye })^{2}$ (Bohr magneton)/kilokayser to the FullRel value, 0.0184 , which is in rather good agreement with the experimental values, 0.014 and 0.0257 . The $A$ term is also improved from -0.2394 $(\text { Debye })^{2}$ (Bohr magneton) in Non-Rel to -0.0756 in Full-Rel. Thus, the relativistic effects are very important for the MCD of molecules containing heavy elements. This change is mainly due to the change in the dipole strength $D$ of ${ }^{1} \mathrm{Q}_{1}$ from 0.4893 (Debye) $^{2}$ in Non-Rel to 0.1257 in Full-Rel. The $A$ / $D$ values of ${ }^{1} \mathrm{Q}_{1}$, which is proportional to the magnetic dipole moment of this state, are -0.49 Bohr magneton in Non-Rel and -0.60 in Full-Rel, respectively, indicating that $A / D$ is nearly invariant as compared to the change of $A$ itself. The $B / D$ values, which can be associated with the magnetic transition moment from this state to others, are 0.40 (Bohr magneton)/kilokayser in Non-Rel and 0.15 in Full-Rel, respectively, indicating that part of the decrease in the $B$ term is attributed to the change in $D$. Although it is difficult to account for the change in $B / D$ because a simple interpretation of the $B / D$ term is unknown, we can say, at least, that the state mixing among ${ }^{1} \mathrm{Q}_{1}$ and triplet states 
causes it. The decrease in $D$ of ${ }^{1} \mathrm{Q}_{1}$ can be explained in terms of the intensity borrowing from ${ }^{1} \mathrm{Q}_{1}$ to the triplet states.

The Faraday terms of the ${ }^{3} \mathrm{Q}_{1}$ and ${ }^{3} \mathrm{Q}_{0}$ states appear only in the relativistic calculations, because these are derived from triplet excitations. The large differences in the experimental properties for ${ }^{3} \mathrm{Q}_{1}$ between [22] and [23] are attributed to the difference in the fitting procedure; the former is based on the best curve fitting of the absorption and MCD spectra, while the latter on the two spectra and the Raman excitation profiles [35] of $\mathrm{CH}_{3}$ I. The calculated $A$ term of ${ }^{3} \mathrm{Q}_{1},-0.0140$ $(\text { Debye })^{2}$ (Bohr magneton), is comparable to the experimental values, -0.0015 and -0.0097 , though it is somewhat overestimated. The $B$ terms of ${ }^{3} \mathrm{Q}_{1}$ and ${ }^{3} \mathrm{Q}_{0}$ states were calculated to be positive and negative, respectively, and the qualitative trend is in accordance with the results by Gedanken et al. However, the absolute values are 0.1778 and
0.1453 (Debye $)^{2}$ (Bohr magneton)/kilokayser for ${ }^{3} \mathrm{Q}_{1}$ and ${ }^{3} \mathrm{Q}_{0}$, respectively, and are much overestimated as compared with the experimental values of 0.0018 and 0.015 by Gedanken et al. and 0.000057 and 0.0222 by Johnson et al.

These overestimations can be attributed to the following two reasons. One is the lack of basis functions with larger exponents that describe the relativistic effects for inner core electrons. In the Full-Rel calculations with Huzinaga's well-tempered Gaussian (WTG) basis, $B$ terms of ${ }^{3} \mathrm{Q}_{1}$ and ${ }^{3} \mathrm{Q}_{0}$ states are 0.0746 and -0.0655 , respectively, and the $A$ term of ${ }^{3} \mathrm{Q}_{1}$ is -0.0025 . Thus, the Faraday terms of ${ }^{3} \mathrm{Q}_{1}$ and ${ }^{3} \mathrm{Q}_{0}$ with the WTG basis are improved as compared with the case of the previous (non-relativistic) basis, though they are underestimated for ${ }^{1} \mathrm{Q}_{1}$. The other is due to electron correlation. The assignments of ${ }^{3} \mathrm{Q}_{1}$ and ${ }^{3} \mathrm{Q}_{0}$ states in the GUHF/SECI calculations are due to the oscillator strengths rather than the excitation en-

Table 2

Analysis by the GUHF/SECI calculations with various relativistic correction levels

\begin{tabular}{|c|c|c|c|c|c|c|c|}
\hline & \multicolumn{5}{|c|}{$\mathrm{GUHF} / \mathrm{SECI}^{\mathrm{a}}$} & \multicolumn{2}{|l|}{ Experimental } \\
\hline & Non-Rel & SFR & SO1 & $\mathrm{SFR}+\mathrm{SO} 1$ & Full-Rel $^{\mathrm{b}}$ & Gedanken et al. ${ }^{\mathrm{c}}$ & Johnson et al. $^{\mathrm{d}}$ \\
\hline $\begin{array}{l}{ }^{3} \mathrm{Q}_{1} \text { state } \\
\text { energy }\end{array}$ & 40252 & 40420 & 39130 & 38999 & 39188 & 33350 & 34900 \\
\hline$A^{\mathrm{e}}$ & 0.0 & 0.0 & -0.0302 & -0.0159 & -0.0140 & -0.0015 & $(-0.0097)^{\mathrm{f}}$ \\
\hline$B^{\mathrm{e}}$ & 0.0 & 0.0 & 0.2343 & 0.2087 & 0.1778 & 0.0018 & -0.000057 \\
\hline$D^{\mathrm{e}}$ & 0.0 & 0.0 & 0.0580 & 0.0298 & 0.0267 & 0.0029 & 0.0193 \\
\hline$f$ & 0.0 & 0.0 & 0.0011 & 0.00055 & 0.00049 & 0.000046 & 0.00032 \\
\hline $\begin{array}{l}{ }^{3} \mathrm{Q}_{0} \text { state } \\
\text { energy }\end{array}$ & 40252 & 40420 & 38127 & 38181 & 38326 & 38300 & 38900 \\
\hline$A^{\mathrm{e}}$ & $\_\mathrm{g}$ & - & - & - & - & - & - \\
\hline$B^{\mathrm{e}}$ & 0.0 & 0.0 & -0.1884 & -0.1735 & -0.1453 & -0.015 & -0.0222 \\
\hline$D^{\mathrm{e}}$ & 0.0 & 0.0 & 0.3487 & 0.4159 & 0.3629 & 0.32 & 0.284 \\
\hline$f$ & 0.0 & 0.0 & 0.0063 & 0.0075 & 0.0065 & 0.0057 & 0.0051 \\
\hline $\begin{array}{l}{ }^{1} \mathrm{Q}_{1} \text { state } \\
\text { energy }\end{array}$ & 48226 & 47908 & 48225 & 47912 & 47850 & 41700 & 40900 \\
\hline$A^{\mathrm{e}}$ & -0.2394 & -0.0937 & -0.1980 & -0.0726 & -0.0756 & -0.044 & $(-0.0377)^{\mathrm{f}}$ \\
\hline$B^{\mathrm{e}}$ & 0.1976 & 0.1264 & 0.0300 & 0.0156 & 0.0184 & 0.014 & 0.0257 \\
\hline$D^{\mathrm{e}}$ & 0.4893 & 0.1915 & 0.3382 & 0.1205 & 0.1257 & 0.088 & 0.0753 \\
\hline$f$ & 0.0111 & 0.0043 & 0.0077 & 0.0027 & 0.0028 & 0.0017 & 0.0014 \\
\hline
\end{tabular}

${ }^{a}$ Huzinaga's uncontracted basis set with exponents determined by a non-relativistic method.

${ }^{\mathrm{b}} \mathrm{SFR}+\mathrm{SO} 1+\mathrm{SO} 2$, actually.

${ }^{\mathrm{c}}[22]$.

$\mathrm{d}$ [23]. Reassignments of the absorption and MCD spectra in [22].

${ }^{\text {e }}$ Units of the excitation energies, $A, B$, and $D$ are same as those in Table 1.

${ }^{\mathrm{f}}$ Assumed the magnetic moment to be 1 Bohr magneton.

${ }^{\mathrm{g}}$ Owing to the non-degeneracy, $A$ terms disappear. 
ergies; the calculated energies are reversed as compared with the experimental ones. This problem will be solved by inclusion of electron correlation. The SAC-CI [28] calculations with perturbation of the SO1 interaction using the same basis set provided the correct order of the excitation energies; 37300, 40200 , and $43500 \mathrm{~cm}^{-1}$ for ${ }^{3} \mathrm{Q}_{1},{ }^{3} \mathrm{Q}_{0}$, and ${ }^{1} \mathrm{Q}_{1}$ states, respectively. Therefore, electron correlation is expected to affect the Faraday terms. Since we do not obtain accurate Faraday terms for the above reason, we cannot judge the validity of the experimental fitting procedures in the present stage. The correlation effects and, in particular, the coupling effects between electron correlation and relativistic effects on MCD are challenging subjects for the future.

Table 2 shows the analysis for the same properties of the three states of $\mathrm{CH}_{3} \mathrm{I}$ : the GUHF/SECI calculations were performed with various relativistic correction levels. The Non-Rel and Full-Rel values in Table 2 are the same as those in Table 1. When the SO1 terms are considered, the Faraday terms of ${ }^{3} \mathrm{Q}_{1}$ and ${ }^{3} \mathrm{Q}_{0}$ appear and the $B$ term of ${ }^{1} \mathrm{Q}_{1}$ is largely improved from 0.1976 to 0.0300 . In addition, the Faraday terms for the SO1, SFR + SO1, and Full-Rel calculations are qualitatively unchanged. Thus, the relativistic effect on MCD is mainly due to the SO1 term in this system. This indicates that the state mixing among the singlet and triplet states is important for the Faraday terms rather than the SFR effect. However, do not mean that the effects due to SFR and $\mathrm{SO} 2$ are negligible. Obviously, the Faraday terms are not additive with respect to the SFR and SO1 terms, and their simultaneous consideration is important. Inclusion of the $\mathrm{SO} 2$ terms results in a relaxation of the effects of SO1 for all of the properties. The trends are consistent with the general behavior of the relativistic corrections due to $\mathrm{SO}$.

\section{Conclusions}

We have developed quasi-relativistic (2-component) generalized unrestricted Hartree-Fock (GUHF)/single excitation configuration interaction (SECI) method and investigated the relativistic effects on the magnetic circular dichroism
(MCD) using the finite perturbation method. The method was applied to the MCD of three $n-\sigma^{*}$ states $\left({ }^{3} \mathrm{Q}_{1},{ }^{3} \mathrm{Q}_{0}\right.$, and ${ }^{1} \mathrm{Q}_{1}$ in Mulliken's notation) of $\mathrm{CH}_{3} \mathrm{I}$. The Faraday terms of the ${ }^{1} \mathrm{Q}_{1}$ state were dramatically improved by the relativistic correction. Although the Faraday terms of the ${ }^{3} \mathrm{Q}_{1}$ and ${ }^{3} \mathrm{Q}_{0}$ states qualitatively reproduced the trends observed by Gedanken et al., the absolute values were much overestimated as compared with the experimental values, and this may be attributed to the effects of the basis set and electron correlations. The relativistic correction was mainly due to the one-electron spin-orbit (SO1) terms. The effects of spin-free relativistic (SFR) and two-electron spin-orbit (SO2) terms were not so large as compared with SO1, though they were not negligible. This indicates that the state mixing among the singlet and triplet states is important for the Faraday terms of this system.

\section{Acknowledgements}

Part of this study has been supported by a Grant-in-Aid for Scientific Research from the Japanese Ministry of Education, Science, and Culture. Y.H. acknowledges a fellowship from the Japan Society for the Promotion of Science (JSPS).

\section{References}

[1] A. Gedanken, O. Schnepp, Chem. Phys. Lett. 37 (1976) 373.

[2] M. Brith-Lindner, S.D. Allen, Chem. Phys. Lett. 47 (1977) 32.

[3] P.A. Snyder, R.W.C. Hansen, E.M. Rowe, J. Phys. Chem. 100 (1996) 17756.

[4] A. Gedanken, O. Schnepp, Chem. Phys. 12 (1976) 341.

[5] I.N. Douglas, R. Grinter, A.J. Thomson, Mol. Phys. 26 (1973) 1257.

[6] S.D. Allen, M.G. Mason, O. Schnepp, P.J. Stephens, Chem. Phys. Lett. 30 (1975) 140.

[7] D.J. Shieh, S.H. Lin, H. Eyring, J. Phys. Chem. 77 (1973) 1031.

[8] A. Kaito, A. Tajiri, M. Hatano, Chem. Phys. Lett. 28 (1974) 197.

[9] Y.H. Yoon, S.T. Lee, D.J. Shieh, H. Eyring, S.H. Lin, Chem. Phys. Lett. 38 (1976) 24.

[10] A.R. Meier, G.H. Wagnière, Chem. Phys. 113 (1987) 287. 
[11] P.J. Stephens, J. Chem. Phys. 52 (1970) 3489.

[12] D.J. Shieh, S.H. Lin, H. Eyring, J. Phys. Chem. 76 (1972) 1844.

[13] S. Coriani, C. Hättig, P. Jørgensen, T. Helgaker, J. Chem. Phys. 113 (2000) 3561.

[14] S. Coriani, P. Jørgensen, A. Rizzo, K. Ruud, J. Olsen, Chem. Phys. Lett. 300 (1999) 61.

[15] E. Goldstein, S. Vijaya, G.A. Segal, J. Am. Chem. Soc. 102 (1980) 6198.

[16] G. Marconi, Chem. Phys. Lett. 146 (1988) 259.

[17] L. Seamans, J. Linderberg, Mol. Phys. 24 (1972) 1393.

[18] J. Michl, Tetrahedron 40 (1984) 3845, Many references are referred to in this review article.

[19] See e.g., J. Fleischhauer, J. Michl, J. Phys. Chem. A 104 (2000) 7776.

[20] H. Nakatsuji, J. Chem. Phys. 61 (1974) 3728.

[21] M. Brith, M.D. Rowe, O. Schnepp, P.J. Stephens, Chem. Phys. 9 (1975) 57.

[22] A. Gedanken, M.D. Rowe, Chem. Phys. Lett. 34 (1975) 39.

[23] B.R. Johnson, J.L. Kinsey, J. Phys. Chem. 100 (1996) 18937.
[24] M.D. Rowe, A. Gedanken, Chem. Phys. 10 (1975) 1.

[25] P. Hafner, W.H.E. Schwarz, Chem. Phys. Lett. 65 (1979) 537.

[26] J. Wan, R. Fukuda, M. Hada, H. Nakatsuji, J. Phys. Chem. A 105 (2001) 128.

[27] R.S. Mulliken, J. Chem. Phys. 8 (1940) 382.

[28] H. Nakatsuji, in: J. Leszczynski (Ed.), Computational Chemistry, Reviews of Current Trends, vol. 2, World Scientific, Singapore, 1997.

[29] B.A. Hess, Phys. Rev. A 33 (1986) 3742.

[30] P.D. Mallinson, J. Mol. Spectrosc. 55 (1975) 94.

[31] S. Huzinaga, J. Andzelm, M. Klobukowski, E. RadzioAndzelm, Y. Sakai, H. Tatewaki, in: Gaussian Basis Set for Molecular Calculations, Elsevier, Amsterdam, 1984.

[32] T.H. Dunning Jr., J. Chem. Phys. 53 (1970) 2823.

[33] S. Huzinaga, M. Klobukowski, Chem. Phys. Lett. 212 (1993) 260.

[34] M. Sugimoto, H. Nakatsuji, J. Chem. Phys. 102 (1995) 285.

[35] G.E. Galica, B.R. Johnson, J.L. Kinsey, M.O. Hale, J. Phys. Chem. 95 (1991) 7994. 